\title{
Antioxidative treatment prevents activation of death-receptor- and mitochondrion-dependent apoptosis in the hearts of diabetic rats
}

\author{
J. Bojunga ${ }^{1}$ D. Nowak ${ }^{2}$ P. S. Mitrou ${ }^{2}$ D. Hoelzer ${ }^{2}$-S. Zeuzem ${ }^{1} \cdot$ K. U. Chow ${ }^{2}$ \\ ${ }^{1}$ Klinik für Innere Medizin II, Saarland University Hospital, Homburg/Saar, Germany \\ ${ }^{2}$ Innere Medizin III, J.W. Goethe-University, Frankfurt am Main, Germany
}

\begin{abstract}
Aims/hypothesis. The mechanisms by which glucose injures cells of the cardiovascular system include generation of reactive oxygen species and induction of cellular apoptosis. To date, little is known about the molecular events of hyperglycaemia-induced apoptosis in the heart in vivo.

Methods. Male Sprague-Dawley rats were rendered diabetic by a single intraperitoneal injection of $60 \mathrm{mg} / \mathrm{kg}$ body weight streptozotocin. Caspase activities in cardiac ventricular tissue were determined using fluorometric and immunoassay caspase-activity assay kits respectively. Expression levels of proteins of the apoptotic cascade were determined with western blot analyses using specific antibodies.

Results. Four weeks of hyperglycaemia induced significant apoptosis in cardiac tissue. Determining the initiators of death-receptor-dependent apoptosis revealed induction of CD95/Fas and caspase-8. Examination of the activities of effector caspases revealed increased activity of caspase- 6 , but not caspase- 3 and
\end{abstract}

-7. On evaluating inhibitors of apoptosis, we found up-regulation of caspase-3 and -7-inhibiting X-linked inhibitors of apoptosis in diabetic rats. Hyperglycaemia also induced significant mitochondrion-dependent apoptosis. Our evaluation of expression levels of Bcl-2 family members showed increased expression of pro-apoptotic Bak and Bax in diabetic rats. Antioxidative treatment with lipoic acid significantly suppressed apoptosis and down-regulated caspase-6, -8 and -9 activity, as well as expression levels of proapoptotic Bcl-2 proteins without changing blood glucose levels.

Conclusions/interpretation. The present study indicates that reactive oxygen species induced by high glucose are involved in both death-receptor- and mitochondrion-dependent apoptosis in the heart in vivo. It also suggests that antioxidants may be a therapeutic option for preventing cardiovascular damage in diabetes mellitus in humans.

Keywords Antioxidants - Apoptosis - Cardiovascular disease - Diabetes - Lipoic acid - Streptozotocin.
Received: 20 May 2004 / Accepted: 18 July 2004

Published online: 10 December 2004

(C) Springer-Verlag 2004

\section{J. Bojunga (®)}

Klinik für Innere Medizin II, Saarland University Hospital,

Kirrberger Straße, 66421 Homburg/Saar, Germany

E-mail: dr_bojunga@web.de

Tel.: +49-6841-1623221, Fax: +49-6841-1623264

Abbreviations: AFC, 7-amido-4-trifluoromethyl-coumarin . IAP, inhibitors of apoptosis · PARP, poly(ADP-ribose) polymerase $\cdot$ ROS, reactive oxygen species $\cdot$ TBST, Tris-buffered saline containing $0.05 \%$ Tween 20

\section{Introduction}

Cardiovascular disease is one of the major complications of diabetes mellitus, resulting in a high percentage of morbidity and mortality and producing significant costs for the healthcare system [1]. The mechanisms of hyperglycaemia-related damage of the cardiovascular system are multifactorial and still not known in detail.

However, there is considerable evidence that hyperglycaemia results in the generation of reactive oxygen species (ROS), leading to increased oxidative stress and cellular damage [2, 3]. Hyperglycaemia 
causes oxidative stress by increasing production of mitochondrial ROS [4], by glucose autoxidation [5] and by non-enzymatic glycation of proteins [6]. In addition, elevated NEFA contribute to increased oxidative stress due to increased mitochondrial uncoupling [7] and beta-oxidation [8]. Increased oxidative stress in turn leads to activation of stress-sensitive, potentially tissue-damaging signalling pathways, including: (i) increased de novo synthesis and accumulation of diacylglycerol and activation of protein kinase $\mathrm{C}$; (ii) increased metabolism of glucose by the sorbitol pathway; (iii) regulation of stress-activated NF- $\mathrm{BB}, \mathrm{p} 38$ MAPK, $\mathrm{NH}_{2}$-terminal Jun kinases/stress-activated protein kinases and AGE/receptor for AGE; (iv) relative or absolute changes in the production of inflammatory mediators and vasoactive substances such as cytokines, prostanoids and nitric oxide [3] respectively; and (v) cellular apoptosis [9].

In the mammalian heart increased apoptosis is seen in conditions such as myocardial infarction, heart failure, hibernation and diabetic cardiomyopathy [10]. Although it is well documented that hyperglycaemia increases oxidative stress [2] and oxidative stress in turn is capable of activating apoptosis signalling pathways [11], little is known to date about the molecular mechanisms of hyperglycaemia-induced apoptosis in the heart in vivo. Some evidence suggests that in hyperglycaemia apoptosis is induced in vitro by activating initiator caspases $[12,13]$. However, hyperglycaemia-induced activation of the death-receptor- and mitochondrion-dependent apoptotic cascade respectively, as well as regulation of inhibitors of apoptosis (IAP) have yet to be determined in vivo.

Hypothesising that long-term hyperglycaemia induces apoptosis signal transduction pathways in diabetes mellitus in vivo, we elucidated markers of death-receptor- as well as mitochondrion-dependent apoptosis in the heart of diabetic rats. In addition, we hypothesised that oxidative stress is one major cause of induction of the apoptosis signal in transduction pathways in diabetes mellitus. To test this, we studied the effects of an antioxidative treatment on markers of apoptosis.

\section{Materials and methods}

Experimental animals. Male Sprague-Dawley rats weighing 250 to $300 \mathrm{~g}$ were purchased from Charles River (Sulzfeld, Germany). They were kept in our laboratory animal facility according to National Institutes of Health standards. Rats were rendered diabetic by a single intraperitoneal injection of $60 \mathrm{mg} / \mathrm{kg}$ body weight streptozotocin (Sigma Chemical, Deisenhofen, Germany) dissolved in $1 \mathrm{ml}$ sodium citrate buffer at $4^{\circ} \mathrm{C}$. Animals were checked for glucosuria with test strips (Diabur; Boehringer, Mannheim, Germany) and blood glucose was measured by the hexokinase method (Clinical System 700 Analyser; Beckman, Munich, Germany). Rats were considered diabetic if they tested positive for glucosuria on 2 consecutive days and the fasting plasma glucose concentration was greater than $11.1 \mathrm{mmol} / \mathrm{l}$. After 4 weeks of hyperglycaemia rats were killed and the heart was removed. Finally, the left ventricle was cut out, washed in $\mathrm{NaCl} 0.9 \%$ solution in order to remove peripheral blood contamination, snap-frozen in liquid nitrogen and stored below $-70^{\circ} \mathrm{C}$ for subsequent protein extraction.

Antioxidative treatment. Antioxidative treatment was carried out using $\alpha$-lipoic acid, a naturally occurring antioxidant [14]. Lipoic acid has been shown to quench free radicals, prevent singlet oxygen-induced DNA damage, exhibit chelating activity, reduce lipid peroxidation, increase intracellular glutathione levels and prevent glycation of serum albumin [14]. As our group has recently shown [15], lipoic acid can reduce oxidative stress, reverse imbalances of inducible nitric oxide synthase and endothelial nitric oxide synthase expression and attenuate increased tissue cyclic GMP activation in the animal model of diabetes used in the present study.

In the treatment group, diabetic rats received intraperitoneal injections of $\alpha$-lipoic acid $100 \mathrm{mg} / \mathrm{kg} 5$ times a week for a period of 4 weeks, starting 5 days after the diagnosis of diabetes.

Caspase activity assays. Fluorometric caspase-activity assay kits (Biocat, Heidelberg, Germany) for caspase-6, -8 and -9 and CaspSELECT caspase immunoassay kits (fluorometric immunosorbent enzyme assay) for caspase-3 and -7 (Biocat) were used for specific and quantitative determination of caspase activity. Tissue homogenates were washed, pelleted and lysed according to the manufacturer's instructions and transferred to 96-well microtitre plates. Samples were then incubated for 2 hours or over night with the supplied, specific 7-amido-4-trifluoromethyl-coumarin (AFC) conjugated caspase substrates (VEID-AFC for caspase-6, IETD-AFC for caspase-8, LEHD-AFC for caspase-9 and DEVD-AFC for caspase-3 and -7). Samples were previously captured by a monoclonal antibody in microtitre plates coated in anti-caspase-3/-7 (100 $\mu \mathrm{l}$ anti-caspase-3/-7 coating solution per well, incubated over night). Through this the substrates were cleaved proportionally to the amount of active caspase in the lysate, generating free AFC, which was measured fluorometrically (excitation wavelength: $400 \mathrm{~nm}$, emission wavelength: $505 \mathrm{~nm}$ ) in a multifunctional reader (Spectrafluor plus Tecan; Crailsheim, Germany). To calculate caspase activity, we used diluted AFC-standards to prepare a six-point calibration curve. First, each absorbance value of all standards was corrected by subtracting the value of the reagent blank, and the mean absorbance value for each standard was determined. Then, analyte concentration was calculated as arbitrary units by correlating its absorbance with the mean absorbance of the standards. The broad-spectrum caspase inhibitor zVAD-fmk (Enzyme Systems Products, Dublin, Calif., USA) was used at a concentration of $50 \mu \mathrm{mol} / \mathrm{l}$ following the manufacturer's instructions.

Western blot analyses. Tissue homogenates were lysed and fractionated by SDS-PAGE (6-15\% gradient gels) and proteins were transferred to a nitrocellulose membrane using an electroblotting apparatus (Bio-Rad, Hercules, Calif., USA) and standard protocols. The membrane was blocked overnight with a $5 \%$ non-fat, dried milk and subsequently incubated with the primary antibody (as specified below). Unbound antibody was removed by washing with Tris-buffered saline $(\mathrm{pH} 7.2)$ containing $0.05 \%$ Tween 20 (TBST). The membrane was then incubated with the secondary antibody (alkaline-phosphataseconjugated antibody; Promega, Madison, Wis., USA) for $2 \mathrm{~h}$ at room temperature. After extensive washing with TBST, proteins were detected upon addition of the development substrates (BCIP; 5-bromo-4-chloro-3-indolyl-phosphate/NBT; 4-nitro-blue-tetrazolium-chloride; Promega). For quantifica- 

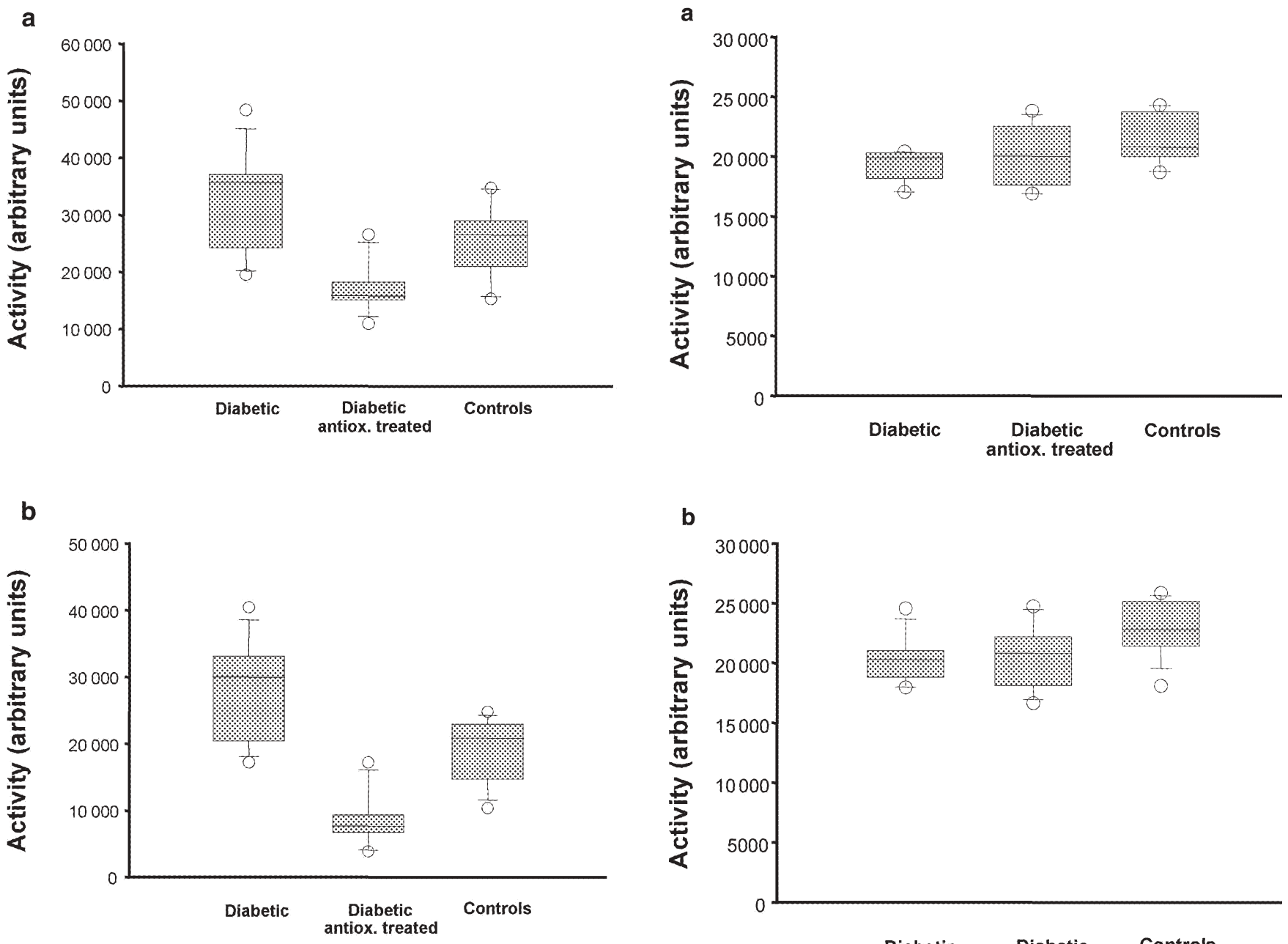

b

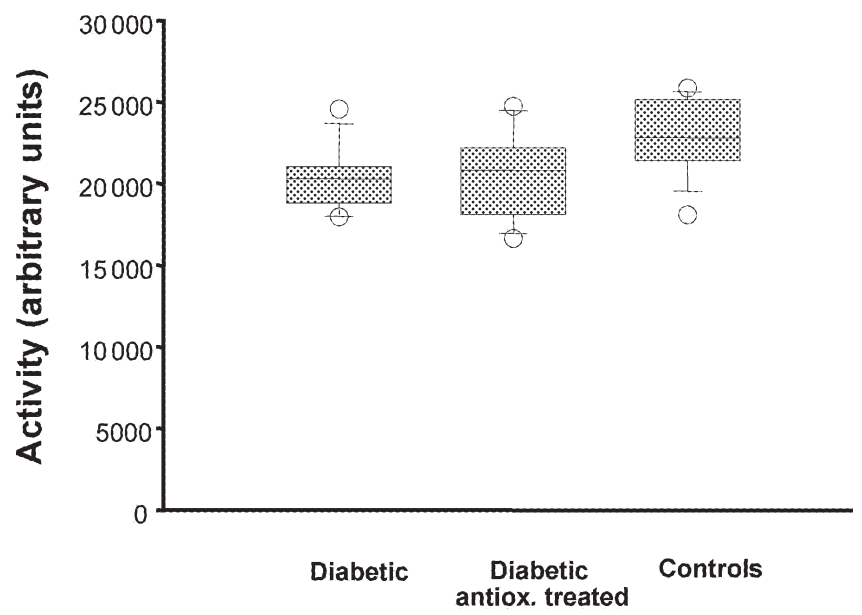

C

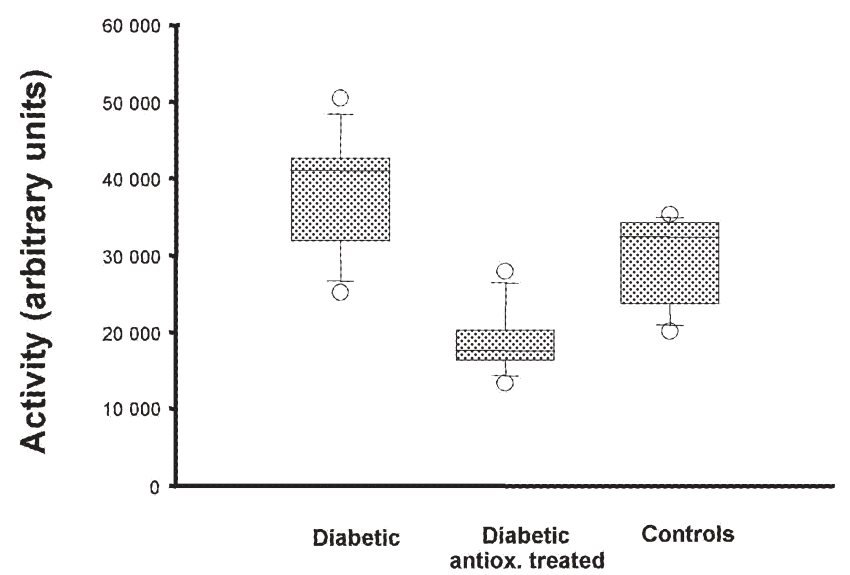

Fig. 2. Activities of caspase-3 (a) and caspase-7 (b) were not significantly affected by long-term hyperglycaemia in the heart of diabetic rats $(n=6)$ compared to controls $(n=6)$. In addition, no significant effect of antioxidative treatment $(n=6)$ on activities of these caspases was found

Antibodies were purchased as follows: for cIAP-1, cIAP-2 and XIAP from R\&D Systems (Minneapolis, Minn., USA); for Bcl-2 from DAKO A/S (Glostrup, Denmark); for Bak, Bax, p53, Daxx from Santa Cruz Biotechnology (Santa Cruz, Calif., USA); for poly(ADP-ribose) polymerase (PARP) from Roche Diagnostics (Mannheim, Germany); and for $\alpha / \beta$-tubulin from Santa Cruz Biotechnology.

Statistics. All statistical operations were performed on a personal computer with the software program SigmaStat (Jandel Scientific). One-way ANOVA with Student-Newman-Keuls test was used to detect significant differences between the groups. Data are expressed as means \pm SEM and a $p$ value of less than 0.05 was considered statistically significant.

\section{Results}

tion, western blot gels were scanned and bands quantified by integrating intensity and area using a commercial computer program (SigmaGel; Jandel Scientific, Erkrath, Germany). Tubulin protein expression served as an internal control.

Metabolic data. Blood glucose concentrations were significantly increased in diabetic rats $(26.2 \pm 2.28$ $\mathrm{mmol} / \mathrm{l} ; n=6$ ) compared to control animals, which had 


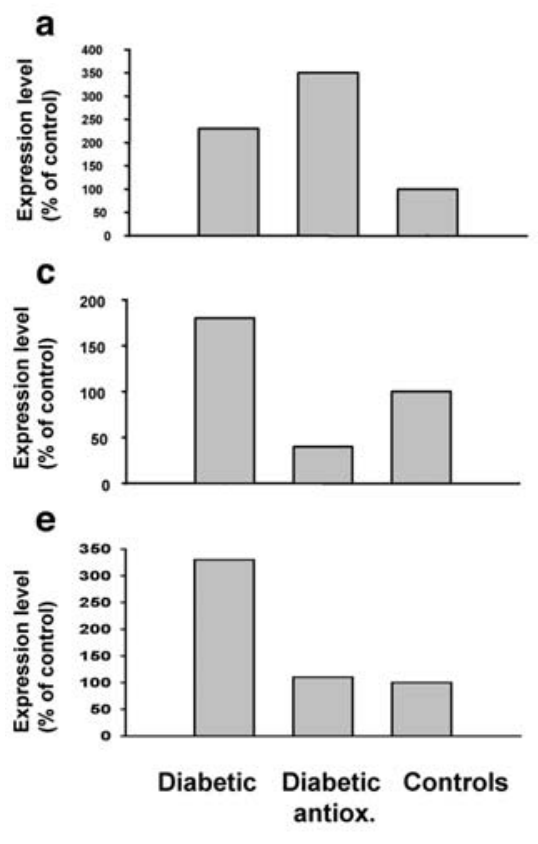

Fig. 3a-g. Expression levels of Bcl-2 family members. Upregulation of anti-apoptotic Bcl-2 (a, b) and pro-apoptotic Bak $(\mathbf{c}, \mathbf{d})$ and $\operatorname{Bax}(\mathbf{e}, \mathbf{f})$ in diabetic rats $(n=6)$ compared to controls $(n=6)$. Antioxidative treatment $(n=6)$ down-regulated expression levels of Bak as well as Bax to the extent found in controls. The highest levels of anti-apoptotic Bcl-2 were detected in the antioxidative-treated group. Tubulin expression (g) served as an internal control

normal non-fasting blood glucose concentrations $(5.99 \pm 0.33 \mathrm{mmol} / \mathrm{l} ; n=6 ; p<0.01)$. Blood glucose concentrations in diabetic rats were not significantly affected by $\alpha$-lipoic acid compared with untreated diabetic rats $(25.36 \pm 1.78 \mathrm{mmol} / \mathrm{l}, n=6$ vs $26.2 \pm 2.28$ $\mathrm{mmol} / \mathrm{l}, n=6 ; p=0.6)$.

Hyperglycaemia increased activities of caspase- $6,-8$ and -9 but not caspase- 3 and -7 , while antioxidative treatment prevented activation of caspase- $6,-8$ and -9 . Hypothesising that long-term hyperglycaemia resulted in activation of caspases, we investigated the activation of caspase-3, -6, -7, -8 and -9 respectively in the hearts of diabetic rats. Compared to control rats, hyperglycaemia led to increased activity of caspase- $6(32,768 \pm 3372 \mathrm{AU}$ vs $25,350 \pm 2106$ AU; $n=6 ; \quad p<0.05), \quad$ caspase- 8 $(28,100 \pm 2861$ AU vs $18,838 \pm 1646$ AU; $n=6 ; p<0.05)$ and caspase-9 $(38,357 \pm 2888$ AU vs $29,463 \pm 1860$ AU; $n=6 ; p<0.05)$. On the other hand, no significant differences in caspase activity were found in caspase-3 $(19,366 \pm 501 \mathrm{AU}$ vs $21,435 \pm 607 \mathrm{AU} ; n=6)$ and caspase$7(20,444 \pm 742$ AU vs $23,040 \pm 777$ AU; $n=6)$. b

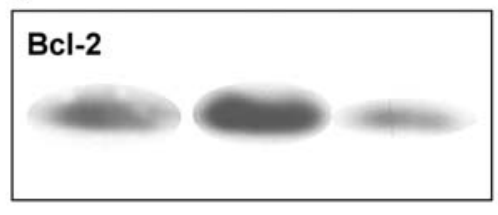

d

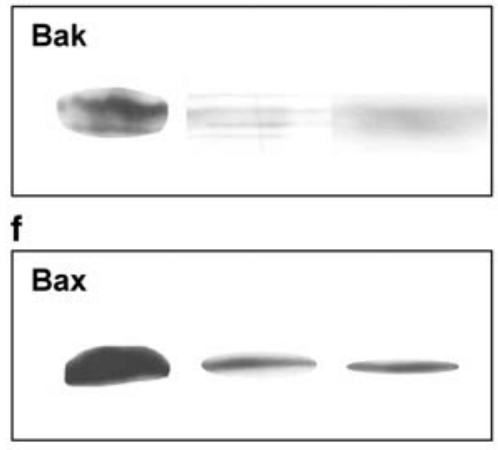

g

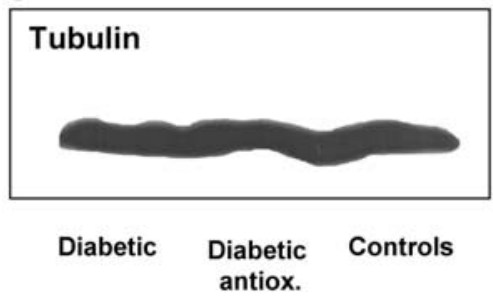

In addition, we hypothesised that oxidative stress may be one major mechanism of caspase activation. In order to test this hypothesis, diabetic rats were treated with antioxidants for 4 weeks. Antioxidative treatment induced a down-regulation of caspase- 6 $(17,275 \pm 1474 ; n=6 ; p<0.05)$, caspase-8 $(8767 \pm 1353$; $n=6 ; \quad p<0.05)$ and caspase-9 $(18,981 \pm 1407 ; n=6$; $p<0.05)$. Interestingly, caspase activity in antioxidative-treated diabetic rats was below that observed in control rats. Activities of caspase- $3(20,105 \pm 844$; $n=6)$ and caspase-7 $(20,724 \pm 883 ; n=6)$ remained unchanged in antioxidative-treated rats compared to control animals as well as untreated diabetic rats. Results for the activity of caspase- $6,-8$ and -9 are summarised in Figure 1, results for caspase- 3 and -7 in Figure 2.

Hyperglycaemia increased expression levels of Bcl-2 family members, while antioxidative treatment prevented increased expression of pro-apoptotic Bcl-2 family members. Bcl-2 members are thought to transmit apoptotic signals and determine their intracellular effects by changes in the expression levels of pro- and anti-apoptotic family members. To elucidate the expression levels of Bcl-2 family members, we analysed Bak, Bax and Bcl-2. Hyperglycaemia $(n=6)$ resulted in an increased expression of pro-apoptotic proteins Bak and Bax. Antioxidative treatment $(n=6)$ in turn downregulated expression levels of Bak as well as Bax to the extent found in control animals $(n=6)$. Expression levels of anti-apoptotic Bcl-2 were increased in diabetic rats compared to control animals, however the 

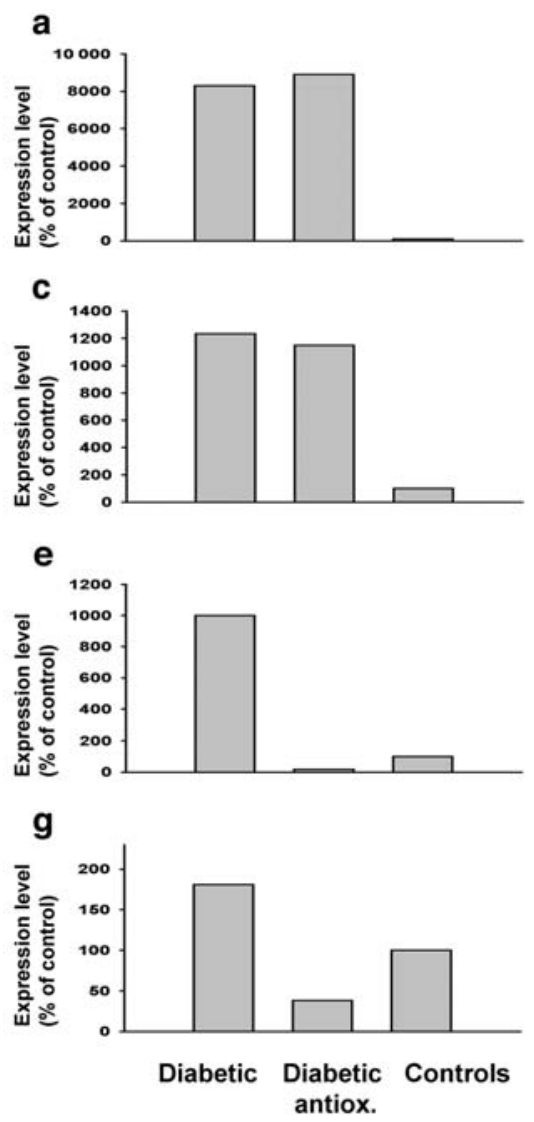

Fig. 4a-i. Expression levels of inductors and markers of apoptosis. Up-regulation of CD95 (a, b), p53 (c, d) and Daxx $(\mathbf{e}, \mathbf{f})$ expression in diabetic rats $(n=6)$. Antioxidative treatment $(n=6)$ completely suppressed expression of Daxx, whereas expression levels of CD95 and p53 remained unchanged. Expression levels of the PARP-1 $(\mathbf{g}, \mathbf{h})$ cleavage product were found in controls $(n=6)$ as well as diabetic animals. Antioxidative treatment suppressed expression levels of the PARP-1 cleavage product. Tubulin expression (i) served as an internal control

highest levels of Bcl-2 were detected in the antioxidative-treated group. Results for $\mathrm{Bcl}-2$ family members are summarised in Figure 3.

Hyperglycaemia increased expression levels of CD95 and p53 without significant effects of antioxidative treatment. Since engagement of CD95 initiates deathreceptor-mediated apoptosis, expression levels of CD95 were assessed. Figure 4 shows that hyperglycaemia ( $n=6$ ) up-regulates CD95 compared to controls $(n=6)$. Interestingly, antioxidative treatment $(n=6)$ did not significantly alter expression levels of CD95.

The nuclear transcription factor p53 regulates transcription of pro-apoptotic genes and causes expression b

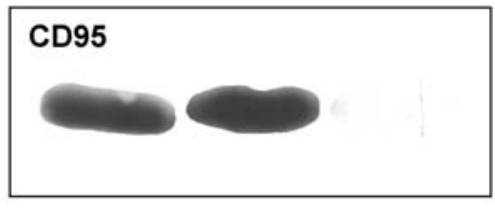

d

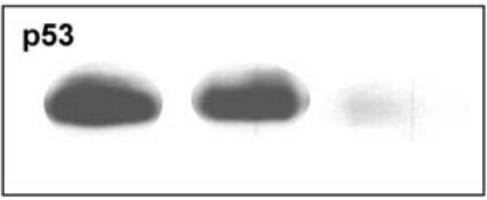

f

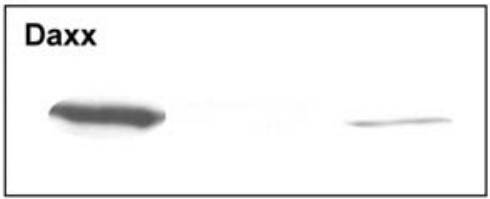

h

$85 \mathrm{Mr}_{\mathrm{r}}$ cleavage product

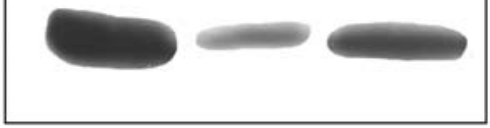

i

Tubulin

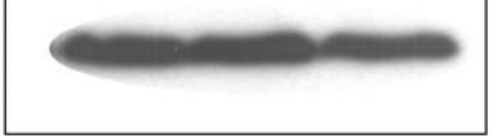

Diabetic Diabetic Controls antiox.

of inhibitors of survival-related proteins. As shown in Figure 4, hyperglycaemia ( $n=6)$ up-regulates expression levels of p53 compared to controls $(n=6)$. Again, antioxidative treatment $(n=6)$ did not significantly alter expression levels of p53.

Hyperglycaemia increased expression levels of Daxx, while antioxidative treatment completely suppressed Daxx expression. In this study, we found up-regulation of Daxx in the heart of diabetic rats $(n=6)$, whereas expression of Daxx was nearly undetectable in control rats $(n=6)$. Antioxidative treatment of diabetic rats $(n=6)$ was associated with a complete suppression of Daxx expression. Results for Daxx are summarised in Figure 4.

Antioxidative treatment suppressed PARP-1 cleavage product expression. Upon activation, caspases cleave numerous cellular proteins, including the DNA repair enzyme PARP. Cleavage of PARP by caspases generates an $85 \mathrm{M}_{\mathrm{r}}$ protein, which is a marker of activated apoptosis. In this study, increased expression levels of the PARP-1 cleavage product were found in diabetic animals $(n=6)$ compared to controls $(n=6)$. Antioxida- 

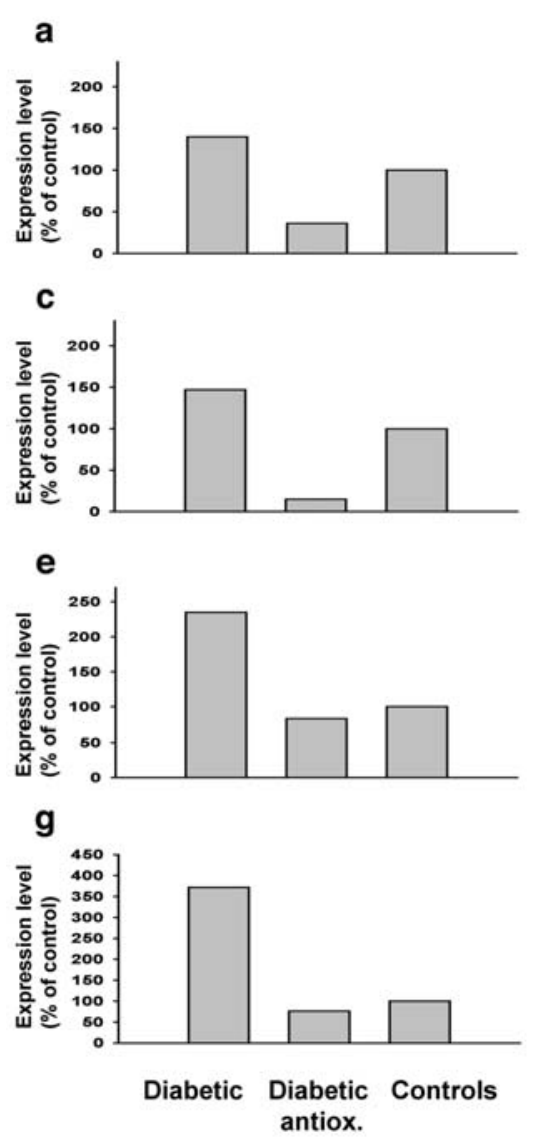

Fig. 5a-i. Expression levels of inhibitors of apoptosis. Whereas expression levels of cIAP-1 (a, b) and cIAP-2 (c, d) were only slightly increased compared to controls $(n=6)$, XIAP $(\mathbf{e}, \mathbf{f})$ and BIR3-RING fragment $(\mathbf{g}, \mathbf{h})$ expression was up-regulated in the heart of diabetic rats. Antioxidative treatment $(n=6)$ was associated with a marked down-regulation of cIAP-1, cIAP-2, XIAP, and of the BIR3-RING fragment. Tubulin expression (i) served as an internal control

tive treatment $(n=6)$ suppressed expression levels of the $85 \mathrm{M}_{\mathrm{r}}$ cleavage product. Results for PARP1/PARP-1 cleavage product are summarised in Figure 4.

Antioxidative treatment suppressed expression levels of inhibitors of apoptosis. IAP proteins confer protection from death-inducing stimuli. Hereby, XIAP, cIAP-1 and cIAP-2 are thought to directly inhibit certain caspases, namely caspase-3, caspase- 7 and caspase-9 [16]. In addition, cleavage of XIAP by caspases generates a protein containing the BIR3-RING subunit. As outlined in Figure 5, hyperglycaemia $(n=6)$ b

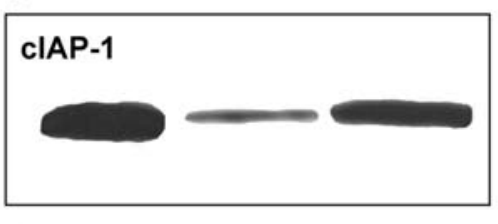

d

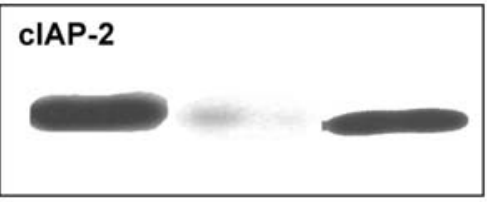

f

XIAP

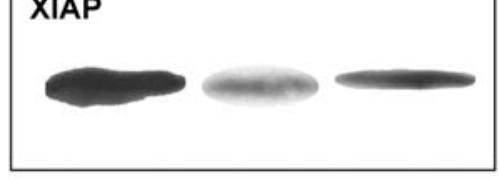

h

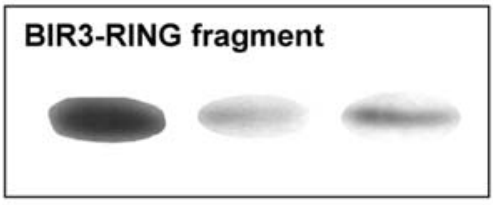

i

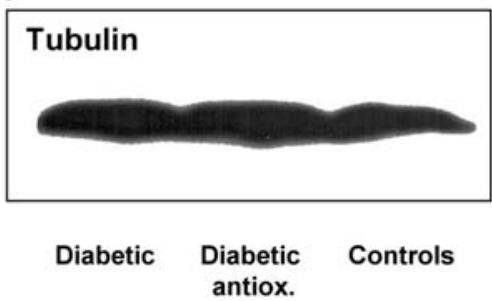

led to an up-regulation of XIAP and the BIR3-RING fragment, whereas expression levels of cIAP-1 and cIAP-2 were only slightly greater than in controls $(n=6)$. Interestingly, antioxidative treatment $(n=6)$ was associated with a marked down-regulation of cIAP-1, cIAP-2, XIAP as well as the BIR3-RING fragment (Fig. 5).

\section{Discussion}

Considerable evidence indicates that increased oxidative stress and induction of apoptosis may play an important role in the development of cardiovascular complications in diabetes mellitus [3]. Numerous studies also indicate that antioxidative treatment may prevent the development of coronary artery disease and diabetic cardiomyopathy $[17,18]$.

Evidence for the existence of apoptosis in the human heart has been reported in various cardiac diseases, including ischaemic and non-ischaemic heart failure, myocardial infarction and arrhythmias [10]. Apoptosis of cardiac myocytes may contribute to pro- 
gressive pump failure, arrhythmias and cardiac remodelling. In experimental diabetes mellitus, the socalled diabetic cardiomyopathy is characterised by cardiac myopathy without coronary heart disease and no other causes of chronic heart failure. Accordingly, cell death by apoptosis is the predominant damage in diabetic cardiomyopathy [19]. Myocyte loss and hypertrophy of the remaining cells characterise the diabetic decompensated heart, mimicking other cardiac myopathies in humans and animals [20]. In one study [20], diabetes progressively depressed ventricular performance in mice and in addition, diabetic mice lost $24 \%$ of their ventricular myocytes and showed a $28 \%$ myocyte hypertrophy.

In the present study, we demonstrated that hyperglycaemia significantly induces apoptosis in the heart of diabetic rats, consistent with earlier reports of myocyte loss in diabetic cardiomyopathy. Furthermore, antioxidative treatment completely suppressed markers of apoptosis, indicating an apoptosis-protecting effect of antioxidative treatment in hyperglycaemia. The antioxidant we used was $\alpha$-lipoic acid, and we chose it because it has recently been shown that some endogenous antioxidants and phase- 2 enzymes in cultured cardiomyocytes can be induced by lipoic acid at low micromolar concentrations in vitro, and that the lipoic-acid-mediated elevation of cellular defences is accompanied by an increased resistance to ROS-elicited cardiac cell injury [21].

To further clarify the molecular basis of increased apoptosis in the heart of diabetic rats, we studied distinct apoptotic signalling pathways, namely deathreceptor- and mitochondrion-dependent apoptosis. We found that in hyperglycaemia death-receptor-dependent apoptosis triggering CD95 was activated, along with significant up-regulation of caspase- 8 , the major initiator caspase in death receptor signalling. Interestingly, while expression levels of CD95 were not affected by antioxidative treatment, activation of caspase- 8 was completely suppressed by antioxidative treatment, even to levels below those found in controls. Our data thus suggest that hyperglycaemia, and as a result oxidative stress are pro-apoptotic stimuli in diabetes mellitus and antioxidative treatment can prevent activation of death receptor signalling, probably at the level of initiator caspases.

To further elucidate signalling pathways of the caspases cascade, we examined the activities of downstream effector caspases, namely caspase-3, caspase-6 and caspase-7 [22]. Somewhat surprisingly, we only found the activity of caspase- 6 to be up-regulated in hyperglycaemia, whereas the activity of caspase- 3 and caspase-7 remained unchanged. In addition, while the activity of caspase- 6 was in turn completely suppressed by antioxidative treatment (again to levels below those found in controls), the activity of caspase- 3 and caspase- 7 was not affected by antioxidative treatment. This suggested that regulation of apoptosis- modifying proteins may be responsible for the observed differences in caspase activities. We therefore determined the expression levels of IAPs, including XIAP, cIAP-1 and cIAP-2, which are potent inhibitors of cell death and also control activation of caspases [23]. Their protective effects are attributed to their ability to directly inhibit certain caspases, namely caspase 3 and caspase 7 respectively [24, 25]. We found that hyperglycaemia led to up-regulation of XIAP, possibly as an autoprotective anti-apoptotic measure of cells undergoing pro-apoptotic stimuli, whereas expression levels of cIAP-1 and cIAP-2 did not differ significantly between diabetic rats and controls. It is noteworthy that antioxidative treatment was associated with a marked down-regulation of cIAP-1, cIAP-2 and XIAP, as well as with the BIR3-RING fragment, reflecting decreased apoptosis and perhaps missing initiation and execution of pro-apoptotic transduction signals due to antioxidative treatment.

Through the cleavage of Bid, caspase- 8 can also activate a second apoptotic pathway, the mitochondrial pathway [26], via the activation of caspase-9 [27]. In this study, caspase-9 activity was significantly increased in the heart of diabetic rats compared to control rats, indicating that mitochondrion-dependent apoptosis was activated in hyperglycaemia. Interestingly, antioxidative treatment completely suppressed caspase-9 activation to levels below that measured in controls, suggesting that oxidative stress plays a crucial role in activating mitochondrion-dependent apoptosis via caspase-9. The events of mitochondrion-dependent apoptosis are controlled by the Bcl-2 family members, which either promote (Bax, Bak, Bad, Bid) or inhibit (Bcl-2, Bcl-xL) apoptosis [28]. In our study, we found a shift towards the expression of the proapoptotic proteins Bak and Bax in hyperglycaemia. Expression levels of anti-apoptotic Bcl-2 were also greater in diabetic rats than in controls, although the highest levels were found in antioxidative-treated rats. Interestingly, antioxidative treatment was associated with a significant down-regulation of pro-apoptotic Bak and Bax, and with an additional up-regulation of anti-apoptotic Bcl-2. Comparable to the activities profiles of initiator caspases, expression levels of proapoptotic Bcl-2 family members were even lower in the antioxidative-treated group than in control animals. Taken together, our data on expression levels of Bcl-2 family members and caspase-9 activity clearly indicate that in hyperglycaemia mitochondrion-dependent apoptosis is activated. They also indicate an apoptosis-protecting effect of antioxidative treatment.

Finally, our study strongly indicates an involvement of p53 in hyperglycaemia-induced apoptosis. Interestingly, antioxidative treatment of diabetic rats did not significantly alter expression of p53 in our experiments. These data are consistent with the results of another study demonstrating that ROS inhibitors failed to protect against activation of p53-mediated apopto- 
sis [29]. This suggests that additional factors beside increased oxidative stress may contribute to the upregulation of p53 expression in hyperglycaemia. Furthermore, the finding of significant caspase-9 regulation in our experiments implies that oxidative stress and in turn antioxidatives may act downstream of p53 activation in this context as described earlier [30].

In 1997, a novel, ubiquitously expressed apoptosis signalling protein, termed Daxx, was described. This protein bound specifically to the Fas death domain [31]. Overexpression of Daxx enhanced Fas-mediated apoptosis downstream of Fas and activated the Jun $\mathrm{N}$-terminal kinase pathway [32]. However, a role of Daxx in hyperglycaemia has not been documented yet. Our experiments suggest an apoptosis-promoting effect of increased Daxx expression, and in addition these findings support the idea that Daxx defines an apoptotic pathway that is downstream of Fas and sensitive to oxidative stress. In addition, because the Daxx apoptotic pathway has been found to be sensitive to Bcl-2 family members [31], it is possible that regulation of $\mathrm{Bcl}-2$ family members with up-regulation of pro-apoptotic Bak and Bax in diabetic rats and up-regulation of anti-apoptotic Bcl-2 in antioxidativetreated diabetic rats may have influenced Daxx expression.

In conclusion, this is the first study demonstrating the molecular events of hyperglycaemia-induced apoptosis in the heart in vivo and indicating that hyperglycaemia induces death-receptor- as well as mitochondrion-dependent apoptosis. Apoptotic myocyte loss could be an important mechanism contributing to progressive dilatation of the heart and development of diabetic cardiomyopathy in humans. Although the mechanistic link can only be shown by directly working with myocytes in culture, the study provides evidence that the main mechanism of hyperglycaemiainduced apoptosis in the heart may be the generation of reactive oxygen species. This may have important implications for the clinical management of diabetes mellitus in humans, and antioxidatives may be an important therapeutic option for preventing cardiovascular damage in diabetes mellitus.

Acknowledgements. This study was supported by grants from the Hecker (to J. Bojunga) and the Adolf Messer foundations (to K.U. Chow), Germany.

\section{References}

1. American Diabetes Association (1998) Economic consequences of diabetes mellitus in the U.S. in 1997. Diabetes Care 21:296-309

2. Baynes JW (1991) Role of oxidative stress in development of complications in diabetes. Diabetes 40:405-412

3. Evans JL, Goldfine ID, Maddux BA, Grodsky GM (2002) Oxidative stress and stress-activating signaling pathways: a unifying hypothesis of type 2 diabetes. Endocr Rev 23:599-622
4. Nishikawa T, Edelstein D, Du XL et al. (2000) Normalizing mitochondrial superoxide production blocks three pathways of hyperglycaemic damage. Nature 404:787-790

5. Wolff SP, Dean RT (1987) Glucose autoxidation and protein modification. The potential role of autoxidative glycosylation in diabetes. Biochem J 245:243-250

6. Wolff SP, Jiang ZY, Hunt JV (1991) Protein glycation and oxidative stress in diabetes mellitus and ageing. Free Radic Biol Med 10:339-352

7. Wojtczak L, Schonfeld P (1993) Effect of fatty acids on energy coupling processes in mitochondria. Biochim Biophys Acta 1183:41-57

8. Yamagishi SI, Edelstein D, Du XL, Kaneda Y, Guzman M, Brownlee M (2001) Leptin induces mitochondrial superoxide production and monocyte chemoattractant protein-1 expression in aortic endothelial cells by increasing fatty acid oxidation via protein kinase A. J Biol Chem 276:25096-25100

9. Chandra J, Samali A, Orrenius S (2000) Triggering and modulation of apoptosis by oxidative stress. Free Radic Biol Med 29:323-333

10. Feuerstein GZ, Young PR (2000) Apoptosis in cardiac diseases: stress- and mitogen-activated signaling pathways. Cardiovasc Res 45:560-569

11. Buttke TM, Sandstrom PA (1994) Oxidative stress as a mediator of apoptosis. Immunol Today 15:7-10

12. Baumgartner-Parzer SM, Wagner L, Pettermann M (1995) High-glucose-triggered apoptosis in cultured endothelial cells. Diabetes 44:1323-1327

13. Ho FM, Liu SH, Liau CS, Huang PJ, Lin-Shiau SY (2000) High glucose-induced apoptosis in human endothelial cells is mediated by sequential activations of c-Jun NH(2)-terminal kinase and caspase-3. Circulation 101:2618-2624

14. Packer LW, Witt EH, Tritschler HJ (1995) Alpha-lipoic acid as a biological antioxidant. Free Radic Biol Med 19:227-250

15. Bojunga J, Dresar-Mayert B, Usadel KH, Kusterer K, Zeuzem S (2004) Antioxidative treatment reverses imbalances of nitric oxide synthase isoform expression and attenuates tissue-cGMP activation in diabetic rats. Biochem Biophys Res Commun 316:771-780

16. Salvesen GS, Duckett CS (2002) IAP proteins: blocking the road to death's door. Nature Rev 3:401-410

17. Rimm EB, Stampfer MJ, Ascherio A (1993) Vitamin E consumption and the risk of coronary heart disease in men. N Engl J Med 328:1450-1456

18. Zhang L, Zalewski A, Liu Y et al. (2003) Diabetes-induced oxidative stress and low-grade inflammation in porcine coronary arteries. Circulation 108:472-478

19. Frustaci A, Kajstura J, Chimenti C et al. (2000) Myocardial cell death in human diabetes. Circ Res 87:1123-1132

20. Kajstura J, Fiordaliso F, Andreoli AM et al. (2001) IGF-1 overexpression inhibits the development of diabetic cardiomyopathy and angiotensin II-mediated oxidative stress. Diabetes 50:1414-1424

21. Cao Z, Tsang M, Zhao H, Li Y (2003) Induction of endogenous antioxidants and phase 2 enzymes by alpha-lipoic acid in rat cardiac $\mathrm{H} 9 \mathrm{C} 2$ cells: protection against oxidative injury. Biochem Biophys Res Commun 310:979-985

22. Slee EA, Adrain C, Martin SJ (2001) Executioner caspase$3,-6$, and -7 perform distinct, non-redundant roles during the demolition phase of apoptosis. J Biol Chem 276: 7320-7326

23. Deveraux QL, Reed JC (1999) IAP family proteins-suppressors of apoptosis. Genes Dev 13:239-252

24. Roy N, Deveraux QL, Takahashi R, Salvesen GS, Reed JC (1997) The c-IAP-1 and c-IAP-2 proteins are direct inhibitors of specific caspases. EMBO J 16:6914-6925 
25. Silke J, Ekert PG, Day CL et al. (2001) Direct inhibition of caspase 3 is dispensable for the anti-apoptotic activity of XIAP. EMBO J 20:3114-3123

26. Kroemer G (2003) Mitochondrial control of apoptosis: an introduction. Biochem Biophys Res Commun 304:433-435

27. Zou H, Li L, Liu X, Wang X (1999) An APAF-1-cytochrome c multimeric complex is a functional poptosome that activates procaspase-9. J Biol Chem 274:11549-11556

28. Reed JC, Jurgensmeier JM, Matsuyama S (1998) Bcl-2 family proteins and mitochondria. Biochim Biophys Acta 1366:127-137

29. Schuler M, Bossy-Wetzel E, Goldstein JC, Fitzgerald P, Green DR (2000) p53 induces apoptosis by caspase activa- tion through mitochondrial cytochrome c release. J Biol Chem 275:7337-7342

30. Johnson TM, Yu ZX, Ferrans VJ, Lowenstein RA, Finkel T (1996) Reactive oxygen species are downstream mediators of p53-dependent apoptosis. Proc Natl Acad Sci USA 93:11848-11852

31. Yang X, Khosravi-Far R, Chang HY, Baltimore D (1997) Daxx, a novel Fas-binding protein that activates JNK and apoptosis. Cell 89:1067-1076

32. Muromoto R, Yamamoto T, Yumioka T et al. (2003) Daxx enhances Fas-mediated apoptosis in a murine pro-B cell line, BAF3. FEBS Lett 540:223-228 